\title{
Observability and coarse graining of consensus dynamics through the external equitable partition
}

\author{
Neave O'Clery, ${ }^{1}$ Ye Yuan, ${ }^{2}$ Guy-Bart Stan, ${ }^{3}$ and Mauricio Barahona ${ }^{1}$ \\ ${ }^{1}$ Department of Mathematics, Imperial College London, London SW7 2AZ, United Kingdom \\ ${ }^{2}$ Department of Engineering, University of Cambridge, Cambridge CB2 1PZ, United Kingdom \\ ${ }^{3}$ Centre for Synthetic Biology and Innovation \& Department of Bioengineering, Imperial College London, London SW7 2AZ, United Kingdom
}

(Received 23 April 2013; published 11 October 2013)

\begin{abstract}
Using the intrinsic relationship between the external equitable partition (EEP) and the spectral properties of the graph Laplacian, we characterize convergence and observability properties of consensus dynamics on networks. In particular, we establish the relationship between the original consensus dynamics and the associated consensus of the quotient graph under varied initial conditions, and characterize the asymptotic convergence to the synchronization manifold under nonuniform input signals. We also show that the EEP with respect to a node can reveal nodes in the graph with an increased rate of asymptotic convergence to the consensus value, as characterized by the second smallest eigenvalue of the quotient Laplacian. Finally, we show that the quotient graph preserves the observability properties of the full graph and how the inheritance by the quotient graph of particular aspects of the eigenstructure of the full Laplacian underpins the observability and convergence properties of the system.
\end{abstract}

DOI: 10.1103/PhysRevE.88.042805

PACS number(s): 89.75.Fb, 89.90.+n

\section{INTRODUCTION}

A current focus of complex systems theory is unravelling the relationship between the topological and spectral properties of a graph, and its dynamics: in particular, the ability of a subset of nodes to reconstruct and manipulate the dynamics on the graph [1-4]. Applications in this area include distributed computing or sensing [5], social or opinion networks [1,6,7], and swarming, flocking or synchronization of coupled oscillators [2,8-11].

A classic setup in this area is the network consensus problem, in which nodes exchange information and come to a common "opinion" or consensus value for a variable [12]. Here we consider the well-known linear consensus framework which operates a nearest-neighbor averaging rule where the dynamics are governed by the Laplacian matrix of the underlying graph $[12,13]$. Linear consensus is often used to model spatially distributed sensors for physical quantities such as position, temperature, voltage, radioactivity or humidity [5], as well as constituting the basis for the design and analysis of flocking and synchronization phenomena $[2,8,14,15]$. There are alternative multiple approaches to modeling such behavior including variations on linear averaging to accommodate timedelay, dynamic, and switching graph topologies as reviewed by $[12,16]$, and gossip or opinion algorithms [17]. Such Laplacian models and their implications in terms of diffusion, community detection, and dynamics are a current focus of research in network theory [18-21].

The external equitable partition (EEP) of a graph is a relaxed version of the well-known equitable partition previously studied in connection with an array of areas including graph coloring [22], and automorphisms [23]. The EEP groups nodes with constant out-degree within each cell (excluding internal edges). The EEP has been shown to be related to consensus dynamics on a graph, including the existence of lower dimensional manifolds defined by the EEP of the graph, as well as cell-averaging properties represented by the quotient graph, a reduction of the original graph in terms of the cells of the EEP $[4,24]$. Recent work has shown that the size of the minimal EEP provides an upper bound on the dimension of the controllable subspace with respect to a controller node $[24,25]$.

The link between the EEP and consensus dynamics is due to the intrinsic relationship between the EEP and the graph Laplacian, and in particular its spectral properties [26]. Here we use and extend the spectral characterization of the EEP to investigate the relationship between the consensus dynamics on the original and quotient graphs under a range of conditions, including asymptotic convergence to the synchronisation manifold under inputs. We also establish how the EEP with respect to a single node can be used to develop a local measure of the asymptotic convergence rate of individual nodes to consensus, as characterized by the second smallest eigenvalue of the quotient Laplacian $[12,13]$.

Furthermore, we investigate the full relationship between the minimal size EEP and the dimension of the observable subspace with respect to an individual node in terms of the spectral properties of the Laplacian "inherited" by the quotient graph associated with the partition. The observable subspace quantifies the ability of the node to determine the state of the entire system from knowledge of its state alone [27,28] and underpins other important properties, such as the minimal number of state values needed for an individual node to compute the consensus value in finite time $[29,30]$. This analysis is also related to its dual controllability problem $[3,27]$.

A brief outline of the paper follows. In Sec. II we introduce notation and define the external equitable partition and corresponding quotient graph. Section III reformulates and extends results linking the EEP to the structure of the eigenspace of the Laplacian matrices of the full and quotient graphs. In Secs. IV and V we investigate the relationship between the consensus dynamics of both the original graph and the quotient graph, and characterize dynamical convergence for nodes with a nontrivial EEP. Finally, in Sec. VI we introduce observability concepts and establish the full relationship between the EEP, observability, and the Laplacian spectrum with respect to an individual node. 


\section{THE EXTERNAL EQUITABLE PARTITION (EEP)}

We consider graphs with $n$ nodes (or vertices) and $e$ edges (or links). The structure of the graph is represented by the adjacency matrix $A$ with entries $A_{i j}$ corresponding to the weight of the directed edge from node $i$ to node $j$. The Laplacian matrix $L$, which characterizes many properties of the graph [31], is given by

$$
L=D-A,
$$

where $D=\operatorname{diag}(A \mathbf{1})$ has the (weighted) node out-degrees on the diagonal. For undirected graphs, $L=L^{T}$ and $L$ is diagonalizable. In this case, the Laplacian matrix is positive semidefinite with all eigenvalues zero or positive, and the graph is connected if the Laplacian has a single zero eigenvalue with corresponding eigenvector $\mathbf{1}$, the vectors of all ones. In the directed case, the Laplacian is not necessarily symmetric but all eigenvalues have semipositive real parts. A directed graph is called strongly connected if and only if any two distinct nodes of the graph can be connected via a path that respects the orientation of the edges, and $\operatorname{rank}(L)=n-1$.

A graph may be partitioned into cells, i.e., groups of nodes $C_{k}=\{h, i, j\}$, where the notation indicates that nodes labeled $h, i$, and $j$ are in cell $k$. A well-known case of a graph partition is the equitable partition, which groups nodes with constant out-degree into cells [32]. The concept of external equitable partition is less restrictive, demanding that the out-degree from nodes in a cell is constant with respect to any other cell but not within each cell.

Definition 1 (External equitable partition (EEP)). A partition $\pi=\left\{C_{1}, \ldots, C_{m}\right\}$ into $m$ cells is said to be externally equitable if each node in $C_{j}$ has the same number of neighbors in $C_{i}$ for all $i, j$ with $i \neq j$.

In the literature, the EEP is alternatively known as the almost equitable partition [26] or the relaxed equitable partition [24]. Clearly, an equitable partition is necessarily an EEP but not vice versa.

We denote the number of cells (or size) of an external equitable partition $\pi$ of the graph as $m=|\pi|$. Clearly, there is always a trivial EEP of size $n$, i.e., every node in a cell by itself. Here we are particularly interested in $\pi^{*}$, the minimal (or coarsest) external equitable partition (mEEP), i.e., the partition with the least number of cells, which is not necessarily unique. The size of $\pi^{*}$ is

$$
m^{*}=\left|\pi^{*}\right|=\min _{|\pi|}\{\pi \mid \pi \text { is } \mathrm{EEP}\} .
$$

A nontrivial mEEP will thus have $m^{*}<n$. We will see below that coarse graining the variables of a consensus dynamics taking place on a graph according to any nontrivial EEP affords us a reduced description of such a dynamics.

Our work also relates local node convergence rates and node observability to the minimal external equitable partition with respect to a single node.

Definition 2 (Minimal EEP with respect to node $r$ ). A partition $\pi_{r}$ with cells $C_{1}, \ldots, C_{m}$ is said to be externally equitable with respect to node $r$ if $\{r\} \in \pi_{r}$. The minimal such partition $\pi_{r}^{*}$ has size

$$
m_{r}^{*}=\left|\pi_{r}^{*}\right|=\min _{\left|\pi_{r}\right|}\left\{\pi_{r} \mid \pi_{r} \text { is EEP and }\{r\} \in \pi_{r}\right\} .
$$

(a)

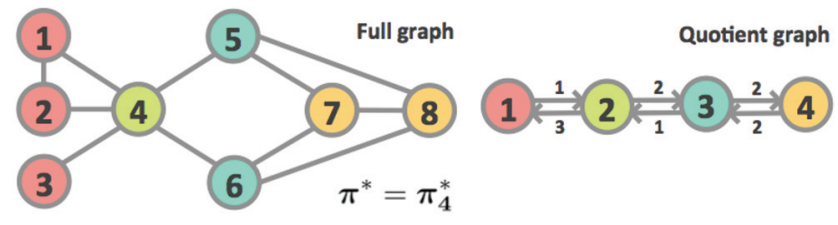

(b)

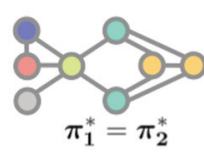

(c)

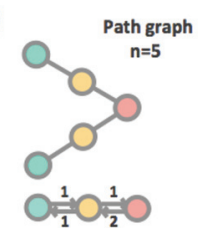

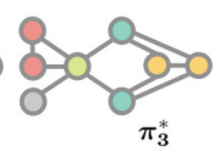

(d)

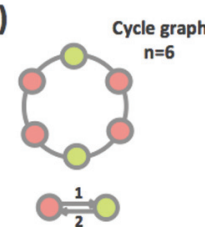

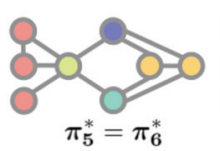

(e)

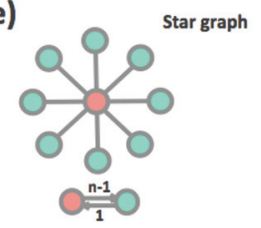

FIG. 1. (Color online) (a) Graph of running Example 1 and its minimal external equitable partition ( $\mathrm{mEEP}), \pi^{*}=\pi_{4}^{*}$ given in Eq. (1), indicated with different shading for each cell. The corresponding quotient graph has directed edges with weights given by the total number of edges connecting the cells in the original graph. (b) The mEEPs with respect to the other nodes of the graph in (a) shown via node shading: $\pi_{1}^{*}=\{\{1\},\{2,3\},\{4\},\{5,6\},\{7,8\}\}$, $\pi_{3}^{*}=\{\{1,2\},\{3\},\{4\},\{5,6\},\{7,8\}\}, \pi_{5}^{*}=\{\{1,2,3\},\{4\},\{5\},\{6\},\{7,8\}\}$, and $\pi_{7}^{*}=\{\{1,2,3\},\{4\},\{5,6\},\{7\},\{8\}\}$. (c)-(e) The mEEP and corresponding quotient graph for (c) the path graph of length $n=5$ : $\mathrm{mEEP}$ of size three, $\pi^{*}=\pi_{3}^{*}$, where the center node is labeled 3 ; (d) the cycle graph of size $n=6$ : mEEP of size two, in which any pair of facing nodes form a cell with the remaining nodes in the other cell; (e) the star graph of size $n$ : mEEP of size two, $\pi^{*}=\pi_{n}^{*}$, where the center node is labeled $n$.

For the case of the mEEP with respect to a single node, it is possible to implement an efficient scheme inspired by algorithms used to find graph automorphisms [23,33]. We have developed such an algorithm to find the mEEP with respect to a single node and applied it to the examples in this paper (see Appendix). A related approach was outlined separately by Cao et al. [34].

Each EEP can be represented through the quotient graph, the associated graph with a node for each cell which is not symmetric in general.

Definition 3 (Quotient graph of an EEP). Given an EEP, the associated quotient graph has a number of nodes equal to the size of the partition (with each node corresponding to a cell of the partition) and directed edge weights given by the out-degree between cells of the partition.

These definitions are illustrated in the following example, which we use as a running example throughout this paper.

Example 1. For the graph in Fig. 1(a), we have searched combinatorically through the whole space of partitions to find the minimal external equitable partition (mEEP):

$$
\pi^{*}=\{\{1,2,3\},\{4\},\{5,6\},\{7,8\}\} .
$$

In addition, we have used our efficient algorithm (Appendix) to find the minimal external equitable partitions with respect 
to each node individually [Figs. 1(a) and 1(b)] with sizes,

$$
m_{r}^{*}=\left\{\begin{array}{lll}
4 & \text { for } & r=4 \\
5 & \text { for } & r=3,5,6,7,8 \\
6 & \text { for } & r=1,2 .
\end{array}\right.
$$

For this example, the mEEP of the full graph is the minimal EEP with respect to node 4: $\pi^{*}=\pi_{4}^{*}$. The corresponding quotient graph is also shown in Fig. 1(a). Figures 1(c)-1(e) also illustrate the mEEP and corresponding quotient graph for a path, cycle, and star graph, respectively, which will be employed as examples in later sections.

In this paper, we will concentrate on partitions of undirected and unweighted graphs, although our framework is equally applicable to undirected weighted graphs. Extensions to general directed graphs for which $L \neq L^{T}$ are also referred to briefly in the discussion section. Note, however, that the quotient graph, which is the coarse-grained graph that results from a partition, will in general be a weighted directed graph.

\section{THE LAPLACIAN SPECTRUM AND THE EEP}

The external equitable partition may also be characterized via the relationship between the Laplacian of the original graph and the Laplacian of the quotient graph. These two Laplacians share spectral properties that underlie the remainder of the analysis in this paper.

Any partition of a graph into $m$ cells can be represented by a characteristic (or indicator) matrix $P \in \mathbb{R}^{n \times m}$, with entries $P_{i j}=1$ if node $i$ is in cell $j$ and zero otherwise. It is also convenient to define the matrix:

$$
D^{\pi}=P^{T} P=\operatorname{diag}\left(\left|C_{i}\right|\right),
$$

where $D^{\pi} \in \mathbb{R}^{m \times m}$ is a matrix with the sizes of the cells $\left|C_{i}\right|$ on the diagonal.

Consider $\pi$, an EEP with indicator matrix $P$. Then the Laplacian matrix of the graph $L$, and the Laplacian matrix of the quotient graph $L^{\pi}$ are related via [26]

$$
L P=P L^{\pi} \text {. }
$$

The relationship given by Eq. (4) translates into specific spectral properties, which we now present and extend in order to give necessary and sufficient conditions for the existence of a nontrivial EEP based on the eigen-structure of the Laplacian.

First, note that Eq. (4) corresponds to the homogeneous Sylvester equation $L P-P L^{\pi}=0$, which has a nonzero solution for $P$ if and only if $L$ and $L^{\pi}$ share at least one eigenvalue [35]. This observation can be extended to characterize the full relationship between the eigenvalues of $L$ and $L^{\pi}$. Let $\sigma(M)$ denote the set of eigenvalues of matrix $M$. If $\pi$ is an EEP, then the eigenvalues of $L^{\pi}$ are a subset of those of $L[26]$ :

$$
\sigma\left(L^{\pi}\right) \subseteq \sigma(L)
$$

A similar statement holds for the eigenvectors: A vector $\mathbf{u}^{\pi}$ is an eigenvector of the quotient graph Laplacian $L^{\pi}$ if and only if $\mathbf{u}=P \mathbf{u}^{\pi}$ is an eigenvector of the graph Laplacian and they share the same eigenvalue [26]. This follows directly from the basic relation given by Eq. (4):

$$
L \mathbf{u}=L P \mathbf{u}^{\pi}=P L^{\pi} \mathbf{u}^{\pi}=\lambda P \mathbf{u}^{\pi}=\lambda \mathbf{u} .
$$

Based on this observation, it was suggested [26] that, if $\mathbf{u}$ is an eigenvector of $L$ corresponding to a shared eigenvalue with $L^{\pi}$, the components of $\mathbf{u}$ must be constant within each cell of $\pi: u_{r}=u_{s}, \forall r, s \in C_{j}$ for $j=1, \ldots, m$. However, this only holds when all eigenvalues of $L$ are distinct.

We now state the results for the general case with repeated eigenvalues. The general proof shows that an eigenvalue of the graph Laplacian is shared with the Laplacian of the quotient graph only if the subspace spanned by the corresponding eigenvectors contains vectors of the form $\mathbf{v}=P \mathbf{v}^{\pi}$ (i.e., with constant coefficients within cells). For the case of nonrepeated eigenvalues, if the components of an eigenvector within each cell are not constant, then the associated eigenvalue is not shared by the quotient graph Laplacian. For the case of repeated eigenvalues, we need to evaluate how many vectors of the form $\mathbf{v}=P \mathbf{v}^{\pi}$ lie in the span of the subspace of the associated degenerate eigenvectors, as can be seen in the following example.

Example 1 continued. For the graph in Fig. 1(a), the Laplacian matrix is

$$
L=\left[\begin{array}{rrrrrrrr}
2 & -1 & 0 & -1 & 0 & 0 & 0 & 0 \\
-1 & 2 & 0 & -1 & 0 & 0 & 0 & 0 \\
0 & 0 & 1 & -1 & 0 & 0 & 0 & 0 \\
-1 & -1 & -1 & 5 & -1 & -1 & 0 & 0 \\
0 & 0 & 0 & -1 & 3 & 0 & -1 & -1 \\
0 & 0 & 0 & -1 & 0 & 3 & -1 & -1 \\
0 & 0 & 0 & 0 & -1 & -1 & 3 & -1 \\
0 & 0 & 0 & 0 & -1 & -1 & -1 & 3
\end{array}\right]
$$

where the lines separate the four cells of the mEEP $\pi^{*}$ given by Eq. (1). Clearly, $\pi^{*}$ is an EEP: The number of edges from any node in cell $i$ to any node in any other cell $j$ is constant, as seen in the off-diagonal blocks.

The quotient graph of $\pi^{*}$, also shown in Fig. 1(a), has indicator matrix $P$ and quotient Laplacian $L^{\pi}$ :

$$
P=\left[\begin{array}{llll}
1 & 0 & 0 & 0 \\
1 & 0 & 0 & 0 \\
1 & 0 & 0 & 0 \\
0 & 1 & 0 & 0 \\
0 & 0 & 1 & 0 \\
0 & 0 & 1 & 0 \\
0 & 0 & 0 & 1 \\
0 & 0 & 0 & 1
\end{array}\right] \text { and } L^{\pi}=\left[\begin{array}{rrrr}
1 & -1 & 0 & 0 \\
-3 & 5 & -2 & 0 \\
0 & -1 & 3 & -2 \\
0 & 0 & -2 & 2
\end{array}\right]
$$

and it is easy to check that $L P=P L^{\pi}$. The set of eigenvalues of the Laplacian $L$ of the full graph is

$$
\sigma(L)=\{0,0.6277,1,3,3,4,4,6.3723\},
$$


and the corresponding eigenvectors are given by the columns of $U$ :

$$
U=\left[\begin{array}{rrrrrrrr}
1 & -1 & -0.5 & -1 & 0 & 0.1667 & 0.1667 & -1 \\
1 & -1 & -0.5 & 1 & 0 & 0.1667 & 0.1667 & -1 \\
1 & -1 & 1 & 0 & 0 & 0.1667 & 0.1667 & -1 \\
1 & -0.3723 & 0 & 0 & 0 & -0.5 & -0.5 & 5.3723 \\
1 & 0.6861 & 0 & 0 & -1 & -0.5 & -0.5 & -2.1861 \\
1 & 0.6861 & 0 & 0 & 1 & -0.5 & -0.5 & -2.1861 \\
1 & 1 & 0 & 0 & 0 & 1 & 0 & 1 \\
1 & 1 & 0 & 0 & 0 & 0 & 1 & 1
\end{array}\right] .
$$

Firstly, note that three of the nonrepeated eigenvalues $\{0,0.6277,6.3723\}$ have eigenvectors with constant components within each cell (columns 1, 2, and 8). Hence these three eigenvalues are shared with the quotient Laplacian. On the other hand, the (nonrepeated) eigenvalue 1 is not an eigenvalue of the quotient graph since its eigenvector (column 3) has nonconstant coefficients in cell 1 . Consider now the degenerate eigenvalues: $\{3,3,4,4\}$. The eigenvectors associated with eigenvalue 3 cannot generate a linear combination that has constant coefficients in each cell. Hence 3 is not an eigenvalue of the quotient graph. On the other hand, the two eigenvectors associated with 4 can generate a linear combination (their sum) with constant coefficients in each cell. Therefore, 4 is an eigenvalue of the quotient Laplacian. In summary,

$$
\sigma\left(L^{\pi}\right)=\{0,0.6277,4,6.3723\},
$$

with eigenvectors given by the columns of $U^{\pi}$ :

$$
U^{\pi}=\left[\begin{array}{rrrr}
1 & -1 & 0.1667 & -1 \\
1 & -0.3723 & -0.5 & 5.3723 \\
1 & 0.6861 & -0.5 & -2.1861 \\
1 & 1 & 0.5 & 1
\end{array}\right] .
$$

The structure of the eigenvectors can be related to whether or not they are shared with the quotient graph Laplacian. If $\mathbf{u}$ is an eigenvector of $L$ with eigenvalue $\lambda$,

$$
L \mathbf{u}=\lambda \mathbf{u}
$$

then the cell-averaged eigenvector,

$$
\mathbf{u}^{\pi}=\left(D^{\pi}\right)^{-1} P^{T} \mathbf{u}
$$

is an eigenvector of the quotient Laplacian $L^{\pi}$ with the same eigenvalue:

$$
\begin{aligned}
L^{\pi}\left(D^{\pi}\right)^{-1} P^{T} \mathbf{u} & =\left(D^{\pi}\right)^{-1} L^{\pi T} P^{T} \mathbf{u}=\left(D^{\pi}\right)^{-1} P^{T} L \mathbf{u} \\
& \Rightarrow L^{\pi} \mathbf{u}^{\pi}=\lambda \mathbf{u}^{\pi},
\end{aligned}
$$

where we have used

$$
P^{T} L P=D^{\pi} L^{\pi}=L^{\pi T} D^{\pi} .
$$

Consequently, if $\mathbf{u}$ is an eigenvector of $L$ and its eigenvalue $\lambda$ is shared with the quotient graph, then it follows from Eq. (8) that the vector that has the cell average as the constant component within each cell,

$$
\mathbf{v}=\left(P\left(D^{\pi}\right)^{-1} P^{T}\right) \mathbf{u}
$$

is also an eigenvector of $L$ with the same eigenvalue:

$$
\begin{aligned}
L\left(P\left(D^{\pi}\right)^{-1} P^{T}\right) \mathbf{u} & =P L^{\pi}\left(D^{\pi}\right)^{-1} P^{T} \mathbf{u}=\lambda\left(P\left(D^{\pi}\right)^{-1} P^{T}\right) \mathbf{u} \\
& \Rightarrow L \mathbf{v}=\lambda \mathbf{v} .
\end{aligned}
$$

This eigenstructure implies that an eigenvector is not shared with the quotient graph if and only if the cellaveraged eigenvector is zero: $\left(D^{\pi}\right)^{-1} P^{T} \mathbf{u}=\mathbf{0}$. For instance, in Example 1 above, multiplication of $U$ by $\left(D^{\pi}\right)^{-1} P^{T}$ leads to cell averaging resulting in all zeros for columns 3,4 , and 5. Hence these columns cannot be associated with shared eigenvalues.

\section{COARSE-GRAINED CONSENSUS DYNAMICS ON THE QUOTIENT GRAPH OF AN EEP}

We now study the implications of the existence of an EEP for network dynamics. As an important example, consider the time evolution of a quantity measured at each node that evolves via information exchange with its neighbors, as represented by the edges of a graph. A simple, widely used model for such network dynamics is the Laplacian-based consensus model [12]. In this simple dynamics, which we define below in Eq. (11), all nodes reach the same fixed (consensus) value asymptotically.

Consider an $n$-dimensional system of ordinary differential equations describing the dynamical evolution of the node variables $\left[x_{1}(t), \ldots, x_{n}(t)\right]^{T} \equiv \mathbf{x}(\mathbf{t}) \in \mathbb{R}^{n}$ :

$$
\dot{\mathbf{x}}(t)=-L \mathbf{x}(t) \text {. }
$$

If the graph is (strongly) connected or, equivalently, its Laplacian has a single zero eigenvalue [12,36], all variables of such a system reach asymptotically a consensus value $x^{*}$ given by

$$
\lim _{t \rightarrow \infty} \mathbf{x}(t)=\frac{\mathbf{w}^{T} \mathbf{x}_{0}}{\mathbf{w}^{T} \mathbf{1}} \mathbf{1} \equiv x^{*} \mathbf{1},
$$

where $\mathbf{w}$ is the left eigenvector of the Laplacian $L$ corresponding to eigenvalue 0 . If the graph is undirected (or directed and balanced; see below), then $\mathbf{w}=\mathbf{1}$ and the consensus value $x^{*}=\mathbf{1}^{T} \mathbf{x}_{0} / n \equiv\left\langle\mathbf{x}_{0}\right\rangle$ is the average of the initial conditions of the variables.

Let the graph have an external equitable partition $\pi$ of size $m$ represented by $P$. We can then consider an $m$ dimensional system $\mathbf{y}(\mathbf{t})=\left[y_{1}(t), \ldots, y_{m}(t)\right]^{T} \in \mathbb{R}^{m}$ defined on the quotient graph and evolving under the dynamics:

$$
\dot{\mathbf{y}}(t)=-L^{\pi} \mathbf{y}(t) \text {. }
$$

Although the quotient graph is in general directed and weighted, it is easy to see that it is strongly connected since the entries $A_{i, j}^{\pi}$ and $A_{j, i}^{\pi}$ are either both nonzero or zero for any pair of nodes $i$ and $j$. Therefore the system defined in Eq. (13) reaches the consensus value $y^{*}$ given by

$$
\lim _{t \rightarrow \infty} \mathbf{y}(t)=\frac{\mathbf{1}^{T} D^{\pi} \mathbf{y}_{0}}{\mathbf{1}^{T} D^{\pi} \mathbf{1}} \mathbf{1}=\frac{\mathbf{1}^{T} D^{\pi} \mathbf{y}_{0}}{n} \mathbf{1} \equiv y^{*} \mathbf{1},
$$

which follows from Eq. (12) with $\mathbf{w}=D^{\pi} \mathbf{1}=$ $\left[\left|C_{1}\right|, \ldots,\left|C_{m}\right|\right]^{T}$.

A special case is when the quotient graph is balanced. A graph is balanced if $\sum_{i} A_{i j}=\sum_{i} A_{j i} \forall j$ (i.e., all nodes have equal number of in- and out-degrees), which implies that the 
leading left eigenvector is $\mathbf{w}=\mathbf{1}$. Clearly, the full graph with $L=L^{T}$ is always balanced and $x^{*}=\left\langle\mathbf{x}_{0}\right\rangle$. If the quotient graph of the EEP is also balanced, which is not always the case, then $y^{*}=\left\langle\mathbf{y}_{0}\right\rangle$, and both the full and quotient dynamics evolve under averaged consensus dynamics [12]. Note that for a balanced quotient graph $D^{\pi} \mathbf{1}=\mathbf{1}$, so that all cells have the same number of elements.

We now examine the dynamical implications of the relationship between the full system given by Eq. (11) and the lower-dimensional system given by Eq. (13) defined by the EEP.

\section{A. EEP consensus is preserved by the full Laplacian dynamics}

Let the full system given by Eq. (11) evolve from an initial condition with constant values within cells: $\mathbf{x}_{0}=P \mathbf{y}_{0}$. Then the system variables remain identical within cells at all times. More explicitly, it follows from Eqs. (11), (13), and (4) that

$$
\mathbf{x}(t)=e^{-t L} P \mathbf{y}_{0}=P e^{-t L^{\pi}} \mathbf{y}_{0}=P \mathbf{y}(t) \forall t,
$$

where $L P=P L^{\pi}$ implies $e^{-t L} P=P e^{-t L^{\pi}}$, and $\mathbf{y}(t)=$ $e^{-t L^{\pi}} \mathbf{y}_{0}$ is the dynamical evolution of Eq. (13) of the consensus on the quotient graph starting with the specified initial condition. Hence, in this case, "cell synchronization" is maintained at all times, and the dynamics of each cell corresponds to that of the corresponding node in the quotient graph. In other words, $\mathbf{x}$ lies in the "cluster synchronization manifold" [37]:

$$
\mathcal{M}^{\pi}=\left\{\mathbf{x} \in \mathbb{R}^{n} \mid x_{i}=x_{j} \forall i, j \in C_{k} \in \pi\right\} .
$$

Finding a nontrivial EEP is thus equivalent to finding a graph (i.e., the quotient graph) for which its associated consensus dynamics describes the dynamics on the lower-dimensional synchronization manifold, which is invariant under the graph Laplacian [37,38].

\section{B. EEP-averaged dynamics evolves under quotient graph Laplacian dynamics}

Let the full system given by Eq. (11) evolve from an initial condition $\mathbf{x}_{0}$ and consider the time evolution of the cell-averaged dynamics:

$$
\langle\mathbf{x}(t)\rangle_{\text {cell }} \equiv\left(D^{\pi}\right)^{-1} P^{T} \mathbf{x}(t) .
$$

Using Eq. (9), it is easy to see that

$$
\begin{aligned}
\langle\mathbf{x}(t)\rangle_{\text {cell }} & =\left(D^{\pi}\right)^{-1} P^{T} e^{-t L} \mathbf{x}_{0}=\left(D^{\pi}\right)^{-1} e^{-t\left(L^{\pi}\right)^{T}} P^{T} \mathbf{x}_{0} \\
& =e^{-t L^{\pi}}\left(D^{\pi}\right)^{-1} P^{T} \mathbf{x}_{0}=e^{-t L^{\pi}}\left\langle\mathbf{x}_{0}\right\rangle_{\text {cell }} .
\end{aligned}
$$

Hence the EEP cell-averaged dynamics of the full system evolves under the Laplacian dynamics of the quotient graph. This averaging relationship, which has also been considered for graph controllability [4,24], holds for the quotient graph of an EEP, and not for an arbitrary partition.

Example 1 continued. Figure 2 shows the evolution of consensus dynamics on the graph in Fig. 1(a) under the full dynamics of Eq. (11) for some $\mathbf{x}_{\mathbf{0}}$. We also show the evolution of Laplacian dynamics given by Eq. (13) on the quotient graph with initial conditions $\mathbf{y}_{\mathbf{0}}=\left(D^{\pi}\right)^{-1} P^{T} \mathbf{x}_{\mathbf{0}}$. The dynamics of the quotient graph correspond to the cell-averaged dynamics of the main graph, and reaches the same consensus value.
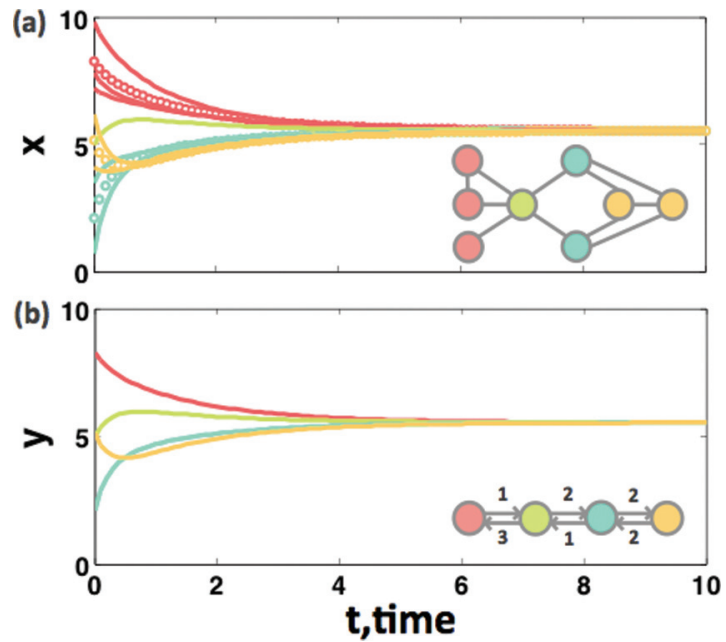

FIG. 2. (Color online) (a) The time evolution of the nodes of the graph of Example 1 (inset) under consensus dynamics defined by Eq. (11) from a random initial condition $\mathbf{x}_{0}$ is plotted with solid lines. The time evolution of $\left(D^{\pi}\right)^{-1} P^{T} \mathbf{x}$, the cell averages according to the mEEP (indicated by the coloring in the inset), are shown with empty circles (for cells of size greater than one). (b) The time evolution of the quotient graph (inset) under consensus dynamics of Eq. (13) with initial condition $\mathbf{y}_{0}=\left(D^{\pi}\right)^{-1} P^{T} \mathbf{x}_{0}$ coincides with that of the cell-averaged dynamics in (a).

It is well known [12] that the convergence rate for consensus dynamics is upper bounded by the second smallest eigenvalue of the symmetric part of the Laplacian [12]. Due to the sharing of eigenvalues between $L$ and $L^{\pi}$ discussed in Sec. III, all the eigenvalues of $L^{\pi}$ are real and the second smallest value of $L^{\pi}$, denoted $\lambda_{2}^{\pi}$, satisfies

$$
\lambda_{2} \leqslant \lambda_{2}^{\pi}
$$

Hence the EEP-averaged dynamics converges at least as fast as the full dynamics. We will show in Sec. $\mathrm{V}$ how this property can be used to characterize the convergence properties of each node through the use of the EEP with respect to individual nodes.

\section{Convergence of consensus dynamics under an input signal consistent with the EEP}

We now consider the consensus dynamics under a smooth input signal $\mathbf{b}(t)$ :

$$
\dot{\mathbf{x}}=-L \mathbf{x}+\mathbf{b}(t) .
$$

The solution of this nonhomogeneous linear system can be written in terms of the eigenvectors $\left(\mathbf{u}_{i}\right)$ and eigenvalues $\left(\lambda_{i}\right)$ of the Laplacian $L$ :

$$
\mathbf{x}(t)=\sum_{i=1}^{n} e^{-\lambda_{i} t}\left(\mathbf{u}_{i}^{T} \mathbf{x}_{0}+\int_{0}^{t} e^{\lambda_{i} s} \mathbf{u}_{i}^{T} \mathbf{b}(s) d s\right) \mathbf{u}_{i} .
$$

If we have an identical input $\mathbf{b}(t)=b(t) \mathbf{1}$, the only surviving integral term corresponds to $\mathbf{u}_{1}=\mathbf{1} / \sqrt{n}$, the eigenvector associated with the zero eigenvalue,

$$
\mathbf{x}(t)=\sum_{i=1}^{n} e^{-\lambda_{i} t}\left(\mathbf{u}_{i}^{T} \mathbf{x}_{0}\right) \mathbf{u}_{i}+\left[\int_{0}^{t} \frac{\mathbf{1}^{T} \mathbf{b}(s)}{n} d s\right] \mathbf{1},
$$


and the system approaches asymptotically a solution where all nodes have identical dynamics:

$$
\mathbf{x}(t) \rightarrow\left[\frac{\mathbf{1}^{T} \mathbf{x}_{0}}{n}+\int_{0}^{t} \frac{\mathbf{1}^{T} \mathbf{b}(s)}{n} d s\right] \mathbf{1} \quad \text { as } t \rightarrow \infty .
$$

The convergence of the system towards global consensus is usually measured by centering the variables with respect to their centroid [30,39]. Let us define the dissensus variables:

$$
\boldsymbol{\delta}=\left(I-\frac{\mathbf{1 1}^{T}}{n}\right) \mathbf{x} \equiv C \mathbf{x},
$$

where $C$ is the centering matrix. The dissensus dynamics evolves according to

$$
\dot{\delta}=-C L \mathbf{x}+C \mathbf{b}(t)=-L C \mathbf{x}+C \mathbf{b}(t)=-L \boldsymbol{\delta}+C \mathbf{b}(t),
$$

where we have used $C L=L=L C$ for an undirected graph with $L=L^{T}$. Clearly, when the inputs are identical,

$$
\mathbf{b}(t)=b(t) \mathbf{1} \Rightarrow \dot{\boldsymbol{\delta}}=-L \boldsymbol{\delta}+b(t) C \mathbf{1}=-L \boldsymbol{\delta},
$$

and the dissensus variables decay exponentially, i.e., all the nodes converge to an identical time-varying solution.

Consider now an EEP defined by $P$. We can define a transformation that measures the dissensus within cells:

$$
\boldsymbol{\delta}^{\pi}=\left(I-P\left(D^{\pi}\right)^{-1} P^{T}\right) \mathbf{x} \equiv C^{\pi} \mathbf{x},
$$

where the variables are referred to the average of their own cell, as follows from Eq. (10). Note that the global centering matrix defined in Eq. (21) is the particular case of $C^{\pi}$ where the partition includes all nodes, i.e., when $P=\mathbf{1}$.

Let the system have an input that is consistent with the EEP, i.e., it is identical within each cell but distinct between them:

$$
\mathbf{b}(t)=P \mathbf{b}^{\pi}(t)
$$

where $\mathbf{b}^{\pi}(t) \in \mathbb{R}^{m \times 1}$ contains the $m$ distinct inputs to the cells. The dynamics of the dissensus within cells for such an input is governed by

$$
\dot{\delta}^{\pi}=-C^{\pi} L \mathbf{x}+C^{\pi} \mathbf{b}(t)=-L C^{\pi} \mathbf{x}+C^{\pi} P \mathbf{b}^{\pi}(t)=-L \boldsymbol{\delta}^{\pi},
$$

where we have used

$$
C^{\pi} L=L C^{\pi},
$$

which follows from Eq. (4) and it is easy to check that $C^{\pi} P=$ 0 . Hence, the dissensus within cells decays exponentially governed by $L$ towards a solution that is uniform within each cell, yet distinct across cells. (Note that there is a constant phase difference in the dynamics on the synchronization manifold fixed by the mean of the arbitrary initial condition.) This conclusion also follows from Eq. (18) by noticing that $P^{T} \mathbf{u}_{i}=0$ for all the eigenvectors of $L$ which are not of the form $\mathbf{u}_{i}=P \mathbf{u}^{\pi}$. Therefore the only terms that survive asymptotically are constant within cells, as shown in the following example.

Example 1 continued. Consider again the graph in Fig. 1(a) with $\pi^{*}$ given in Eq. (1) and indicator matrix $P$ as in Eq. (5). Let the driven system of Eq. (17) evolve under $\mathbf{b}(t)=P \mathbf{b}^{\pi}(t)$, an input consistent with this EEP. Figure 3(b) shows that the dynamics of Eq. (17) converges to a time-varying solution with $m=4$ distinct trajectories from a random initial condition $\mathbf{x}_{0}$.
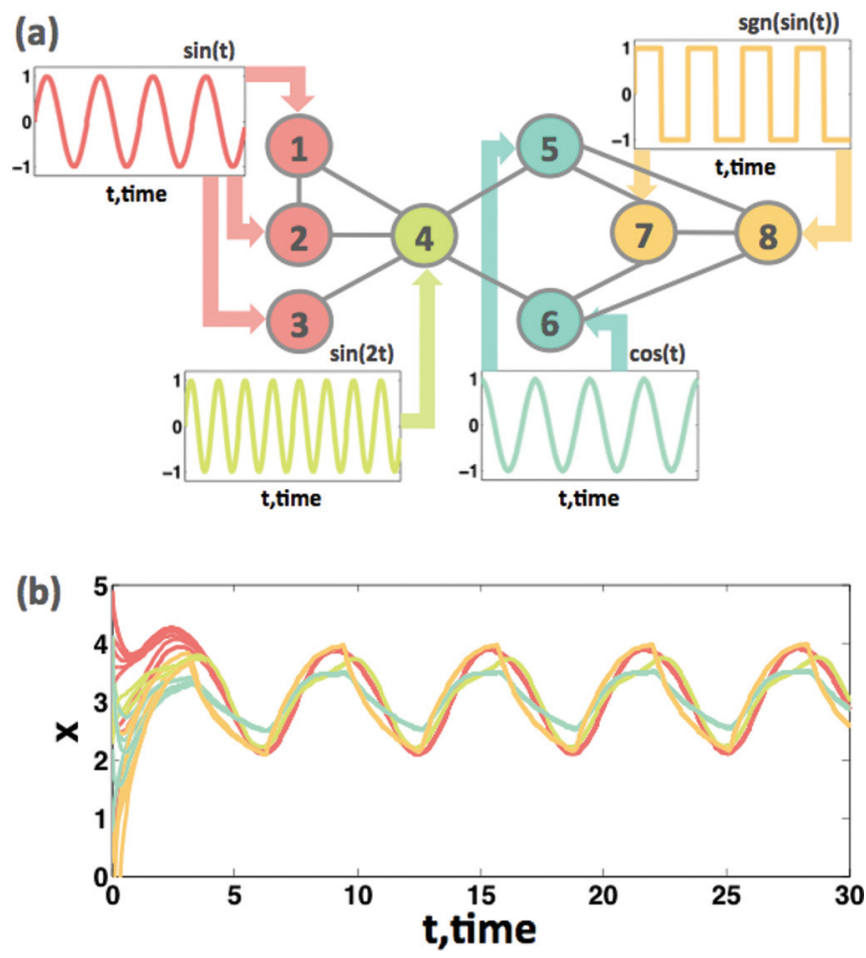

FIG. 3. (Color online) (a) The graph of Example 1 with input $\mathbf{b}(t)=P \mathbf{b}^{\pi}(t) \equiv P[\sin (t), \sin (2 t), \cos (t), \operatorname{sgn}(\sin (t))]^{T}$. (b) For several random initial conditions (with the same mean so that they have identical constant phase), the dynamics asymptotically converges to the synchronization manifold with four distinct time-varying outputs, one for each cell.

\section{NODE CONVERGENCE AND THE EEP WITH RESPECT TO A SINGLE NODE}

We now show how the mEEP with respect to each node allows us to characterize node convergence when the network evolves under consensus dynamics. As discussed in Sec. II, $m E E P$ with respect to each node considers partitions in which the node under study is in a cell by itself. Finding the mEEP with respect to a single node is computationally tractable through the use of a recursive partitioning algorithm inspired by search schemes for graph isomorphisms. We have developed such an algorithm and applied it to characterize node properties of the graphs below. See Appendix for a brief summary of the code.

Our analysis of the spectral properties of the EEP (Sec. IV) shows that the rate of convergence of the EEP dynamics is at least as fast as that of the original dynamics, as follows from Eq. (16). Hence the EEP with respect to each node $\left(\pi_{r}\right)$ can be used to characterize the convergence properties of individual nodes, since the rate of convergence of variable $x_{r}$ is upper bounded not by $\lambda_{2}$ but by $\lambda_{2}^{\pi_{r}}$. In particular, we will be interested in finding nodes $r$ for which

$$
\lambda_{2} \ll \lambda_{2}^{\pi_{r}},
$$

i.e., the spectral gap of the EEP quotient graph $\left(\lambda_{2}^{\pi_{r}}\right)$ is larger than that of the full graph $\left(\lambda_{2}\right)$. When this is the case, the rate of convergence of the node will be faster than that of the rest of the graph. 
Example 1 continued. Consider again the graph in Fig. 1(a). Using our efficient search algorithm (Appendix) to find the mEEPs with respect to each node in turn, we find that $\lambda_{2}^{\pi_{r}^{*}}=\lambda_{2}$ for all $\pi_{r}^{*}$. Hence the individual nodes do not exhibit differentiated rates of convergence, as can be seen in Fig. 2.

However, other graphs can have specific spectral properties which imply that particular nodes have distinct convergence rates to the rest of the graph, as can be seen through the following examples.

Star graph. Consider the consensus dynamics on a star graph with $n$ nodes, where the central node is labeled $n$ [the inset in Fig. 4(a) shows an example with $n=8$ ]. An orthogonal set of Laplacian eigenvectors $\mathbf{u}_{i}$ are given by the columns of

$$
U=\left[\begin{array}{rrrccc}
1 & 1 & 0 & \ldots & 0 & -\frac{1}{n-1} \\
1 & -1 & 1 & \ldots & 0 & -\frac{1}{n-1} \\
1 & 0 & -1 & \ldots & 0 & -\frac{1}{n-1} \\
\vdots & \vdots & \vdots & \ddots & \vdots & \vdots \\
1 & 0 & 0 & \ldots & 1 & -\frac{1}{n-1} \\
1 & 0 & 0 & \ldots & -1 & -\frac{1}{n-1} \\
1 & 0 & 0 & \ldots & 0 & 1
\end{array}\right],
$$

corresponding to eigenvalues $\lambda_{1}=0 ; \lambda_{i}=1$ for $i=$ $2, \ldots, n-1 ;$ and $\lambda_{n}=n[40]$.

The mEEP with respect to the center node is $\pi_{n}^{*}=$ $\{\{1, \ldots, n-1\},\{n\}\}$. For this partition, $\left(D^{\pi_{n}^{*}}\right)^{-1} P^{T} \mathbf{u}_{i}=$ $[0,0]^{T}$ for all the degenerate eigenvectors $i=2, \ldots, n-1$ and only $\lambda_{1}=0$ and $\lambda_{n}=n$ are shared with the quotient graph Laplacian. Hence $\lambda_{2}=1<\lambda_{2}^{\pi_{n}^{*}}=n$ and the center node converges more quickly than the full system [Fig. 4(a)].

On the other hand, the mEEP with respect to any of the leaf nodes is $\pi_{r}^{*}=\{\{1, \ldots, r-1, r+1, \ldots, n-1\},\{r\},\{n\}\}$ for $r=1, \ldots, n-1$. In this case, there is one linear combination of the degenerate eigenvectors that has constant components within each cell. Therefore the eigenvalue 1 is also shared with the quotient graph and $\lambda_{2}^{\pi_{r}^{*}}=\lambda_{2}=1$ implying that the convergence rate is not faster than for the full graph [Fig. 4(c)].

Path graph. Consider a path graph of length $n$ with Laplacian eigenvalues and eigenvectors $\left\{\lambda_{i}, \mathbf{u}_{i}\right\}_{i=1}^{n}$ given by [41]

$\lambda_{i}=2-2 \cos \left(\frac{\pi(i-1)}{n}\right)$,

$\mathbf{u}_{i}=\left[\ldots, \cos \left(\frac{\pi(i-1)(2 j-1)}{2 n}\right), \ldots\right]^{T}, \quad j=1, \ldots, n$.

Let $n$ be odd and consider the mEEP with respect to the center node $r=(n+1) / 2: \pi_{r}^{*}=\{\{1, n\},\{2, n-1\}, \ldots,\{(n-1) / 2$, $(n+3) / 2\},\{(n+1) / 2\}\}$. An example with $n=5$ is shown in Fig. 1(c). The center coordinate of the second eigenvector is zero, $u_{2,(n+1) / 2}=0$, and the rest of the components are antisymmetric with respect to the center: $u_{2, j}=-u_{2,(n-j)}$ for $j=1, \ldots,(n-1) / 2$. Hence $\left(D^{\pi}\right)^{-1} P^{T} \mathbf{u}_{2}=\mathbf{0}$, and $\lambda_{2}$ is not shared with the quotient graph. Due to the same antisymmetry, $\left(D^{\pi}\right)^{-1} P^{T} \mathbf{u}_{i}=\mathbf{0}$ for $i=4,6, \ldots, n-1$, and none of the corresponding eigenvalues (with even indices) is shared with the quotient graph. On the other hand, the odd eigenvectors are symmetric about the center node: $u_{i, j}=u_{i, n-j}$ for $j=$
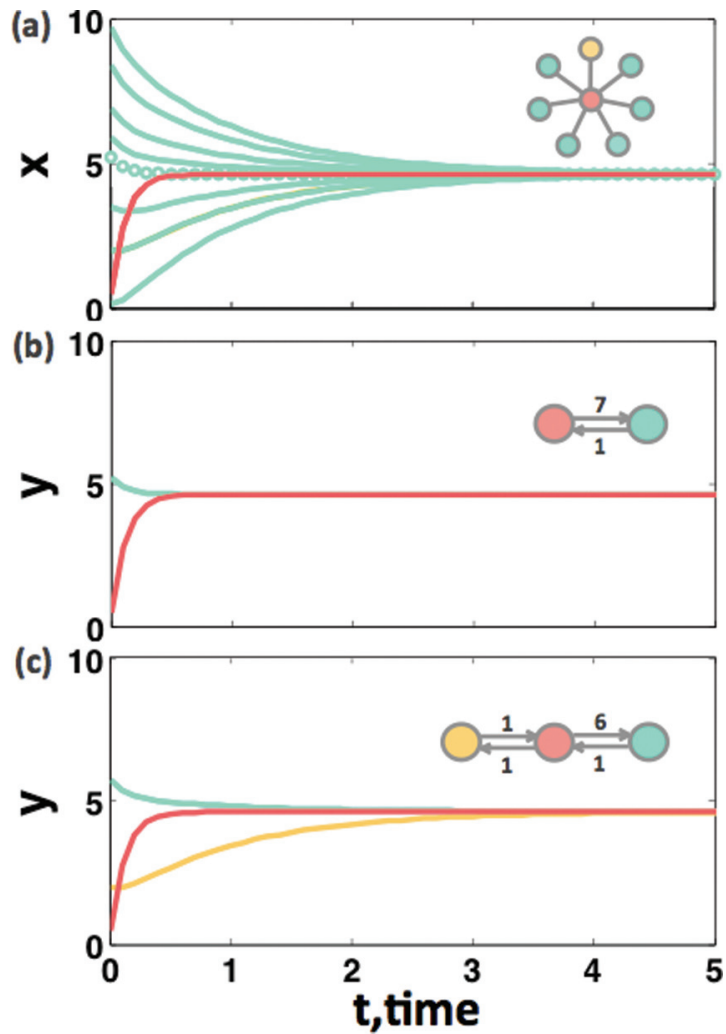

FIG. 4. (Color online) (a) Consensus dynamics of a star graph with $n=8$ nodes (inset) starting from a random initial condition $\mathbf{x}_{0}$. The time-dependent average of all the spoke nodes (including the [orange (medium gray)] top spoke node) is shown with empty circles. (b) Consensus dynamics of the quotient graph (inset) of the mEEP of the star graph with respect to the center node $n$ starting from the initial condition $\mathbf{y}_{0}=\left(D^{\pi_{n}^{*}}\right)^{-1} P^{T} \mathbf{x}_{0}$. The dynamics exhibits fast convergence, upper bounded by $\lambda_{2}^{\pi_{n}}=8 \gg \lambda_{2}=1$. (c) Consensus dynamics of the quotient graph (inset) of the mEEP of the star graph with respect to any leaf node $i$ for $i=1, \ldots, n-1$ (e.g., [orange (medium gray)] top node), again with $\mathbf{y}_{0}=\left(D^{\pi_{i}^{*}}\right)^{-1} P^{T} \mathbf{x}_{0}$. In this case, $\lambda_{2}^{\pi_{i}^{*}}=\lambda_{2}=1$ and leaf nodes do not exhibit faster convergence.

$1, \ldots,(n-1) / 2$, and $i=3,5, \ldots, n$, and these eigenvectors are all shared with the quotient graph:

$$
\sigma\left(L^{\pi_{r}^{*}}\right)=\left\{\lambda_{2 i-1}, i=1, \ldots,(n+1) / 2\right\}
$$

In this case, $\lambda_{2}^{\pi_{r}^{*}}=\lambda_{3}$ and the center node converges faster, as seen in Fig. 5 .

Using the same analysis, one can show that for any noncentral node of a path with $n$ odd, all the eigenvectors are inherited by the quotient graph. Hence $\left|\pi_{r}^{*}\right|=n, \forall r \neq$ $(r+1) / 2$, and there is no increase of the spectral gap of the $\mathrm{mEEP}$. The convergence of noncentral nodes is therefore just as fast as the full graph.

For paths with $n$ even, it follows from similar symmetry arguments that all eigenvectors are inherited by the quotient graph and $\left|\pi_{r}^{*}\right|=n, \forall r$. Hence $\lambda_{2}^{\pi_{r}^{*}}=\lambda_{2}$ and there are no fastconverging nodes in such graphs. 


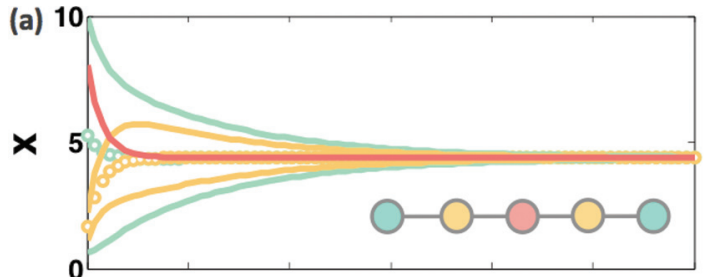

(b)

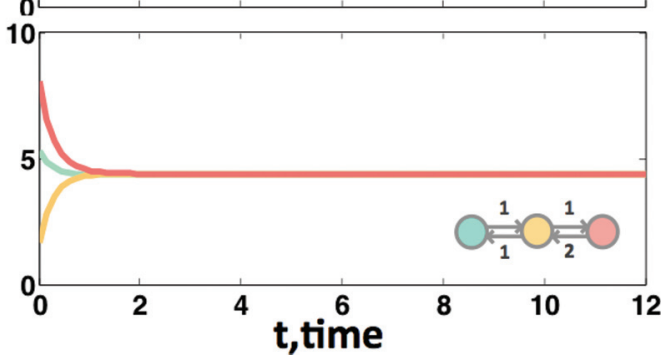

FIG. 5. (Color online) (a) Consensus dynamics of a path graph with $n=5$ nodes (inset) starting from a random initial condition $\mathbf{x}_{0}$. The time-varying averages within cells $\{1,5\}$ and $\{2,4\}$ are shown with empty circles. (b) Consensus dynamics of the quotient graph (inset) corresponding to the mEEP of the path with respect to the center node $r=3$ [red (medium gray)]. The dynamics is started from the initial condition $\mathbf{y}_{0}=\left(D^{\pi}\right)^{-1} P^{T} \mathbf{x}_{0}$ and exhibits fast convergence, upper bounded by $\lambda_{2}^{\pi_{3}}=1.382>\lambda_{2}=0.382$. Node 3 is therefore fast converging.

\section{OBSERVABILITY OF CONSENSUS DYNAMICS AND THE EEP}

\section{A. Node observability of consensus dynamics}

The external equitable partition with respect to a single node is related to the observability of the graph from a single observed node. We now introduce briefly the concept of graph observability from a linear systems perspective.

A dynamical system is said to be observable if the state of the system may be uniquely determined by the evolution of the observed node(s). Thus observability is a measure of how well the internal states of a system can be inferred from its observed outputs [27]. Consider a graph with $n$ nodes evolving under the consensus dynamics of Eq. (11). We then define the observability matrix with respect to node $r$ :

$$
\mathcal{O}_{r}=\left[\begin{array}{c}
\mathbf{e}_{r}^{T} \\
\mathbf{e}_{r}^{T}(-L) \\
\mathbf{e}_{r}^{T}(-L)^{2} \\
\vdots \\
\mathbf{e}_{r}^{T}(-L)^{n-1}
\end{array}\right] \in \mathbb{R}^{n \times n}
$$

where $\mathbf{e}_{r} \in \mathbb{R}^{n}$ is the unit vector with a 1 in position $r$ and zeros elsewhere. A fundamental result in control theory [27,29] establishes that the consensus system given by Eq. (11) is $r$ observable if and only if $\mathcal{O}_{r}$ is full rank. Then the observations of node $r$ can uniquely determine the entire initial state $\mathbf{x}_{0}$ of the system.

In general, we are interested in characterizing the dimension of the r-observable subspace given by the rank of the matrix $\mathcal{O}_{r}$. This quantity is useful for a variety of applications, including the characterization of the minimal number of state values
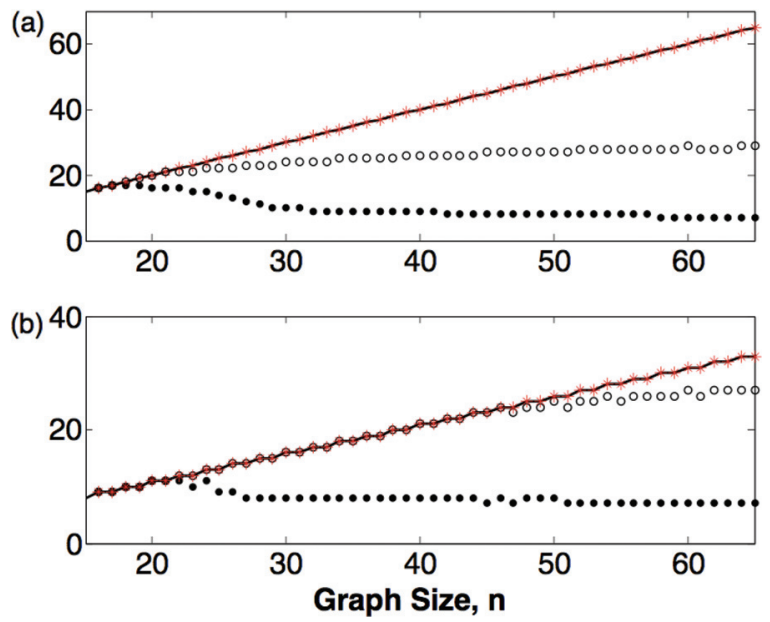

FIG. 6. (Color online) Numerical instability of rank algorithms for observability matrices: (a) The observability of a path graph of size $n$, where the observability with respect to node 1 (or equivalently node $n$ ) is $\operatorname{rank}\left(\mathcal{O}_{1}\right)=m_{1}^{*}=n, \forall n$ (top solid line) (30) and (31). The standard MATLAB rank function (solid circles) becomes numerically unstable for $n \geqslant 18$, while an improved pivoted version (open circles) also becomes unstable for $n \geqslant 22$. Our algorithm based on Eq. (27) (asterisks) recovers the true result (i.e., the asterisks lie on the solid line) with no numerical instability. (b) Same as (a) for a cycle graph of size $n$, where $\operatorname{rank}\left(\mathcal{O}_{r}\right)=m_{r}^{*}=\lceil(n+1) / 2\rceil$.

needed for an individual node to compute the consensus value of a system in finite time $[29,30,42]$. However, the numerical calculation of the rank of a matrix, usually based on the singular value decomposition, can be highly unstable even for observability matrices of relatively small dimensions (Fig. 6).

A significantly more stable rank computation follows from the following decomposition that takes advantage of the eigenstructure of the Laplacian:

$$
\operatorname{rank}\left(\mathcal{O}_{r}\right)=|S|-\left|V_{r}\right|,
$$

where $S$ is the set of distinct eigenvalues of $L$ and

$$
V_{r}=\left\{\lambda \mid L v_{i}=\lambda v_{i} \text { and } v_{i}(r)=0, \forall i=1, \ldots, \operatorname{multi}(\lambda)\right\},
$$

with multi $(\lambda)$ the (algebraic) multiplicity of eigenvalue $\lambda$. To obtain Eq. (27), consider the diagonalization:

$$
-L=U \Lambda U^{-1} \text {, }
$$

where $U$ has the eigenvectors for columns, and $\Lambda$ is the diagonal matrix of eigenvalues. From Eq. (26), we can then derive that

$$
\mathcal{O}_{r}=\left[\begin{array}{c}
\mathbf{u}_{r} \\
\mathbf{u}_{r} \Lambda \\
\mathbf{u}_{r} \Lambda^{2} \\
\vdots \\
\mathbf{u}_{r} \Lambda^{n-1}
\end{array}\right] U^{-1}=H \operatorname{diag}\left(u_{r, i}\right) U^{-1},
$$

where $\mathbf{u}_{r}=\mathbf{e}_{r}^{T} U$ is a $1 \times n$ vector that contains the $r$ th component of the eigenvectors of $L$, and $H$ is the Vandermonde matrix [43] of the eigenvalues with entries $H_{i, j}=\lambda_{j}^{i-1}$. Since $L$ is diagonalizable, it immediately follows that $U^{-1}$ is full 
rank and

$$
\operatorname{rank}\left(\mathcal{O}_{r}\right)=\operatorname{rank}\left(H \operatorname{diag}\left(u_{r, i}\right)\right)<|S|,
$$

where the inequality follows from the fact that the rank of the Vandermonde matrix $H$ is given by the number of distinct eigenvalues of $L$. Since the entries of the vector $\mathbf{u}_{r}$ multiply the corresponding column of $H$, the only way in which the product $H \operatorname{diag}\left(u_{r, i}\right)$ can drop rank further is when one or more of the $r$ th components are zero. Hence Eq. (27) follows.

Equation (27) is related to the classic Popov-BelevichHautus $(\mathrm{PBH})$ lemma in control theory and related results on partial observability and the grounded Laplacian [30]. Note also that this result applies to any diagonalizable Laplacian, even if it is nonsymmetric. This is precisely the case of the Laplacian of the quotient graph and we will apply Eq. (27) to characterize fully the connection of the mEEP and $r$ observability in Sec. VI B below.

As the numerics in Fig. 6 show, we can use Eq. (27) as the basis for a stable algorithm to compute the dimension of the $r$-observable subspace for consensus dynamics on graphs. We have applied this algorithm throughout the rest of this paper.

Example 1 continued. For our main example in Fig. 1(a), the number of distinct eigenvalues in Eq. (6) is $|S|=6$ and from the zeros in the components of the eigenvectors of Eq. (7), it follows that

$$
\operatorname{rank}\left(\mathcal{O}_{r}\right)=|S|-\left|V_{r}\right|=\left\{\begin{array}{lll}
4 & \text { for } & r=4,7,8 \\
5 & \text { for } & r=3,5,6 \\
6 & \text { for } & r=1,2
\end{array}\right.
$$

In addition to our running Example 1, we can use the results in Sec. V to conclude that

(1) Star graph. It is easy to see from Eq. (24) that $\operatorname{rank}\left(\mathcal{O}_{n}\right)=2 \operatorname{and} \operatorname{rank}\left(\mathcal{O}_{r}\right)=3, \forall r \neq n$, where $n$ is the center node.

(2) Path graph. We saw above that

$$
m_{r}^{*}= \begin{cases}(n+1) / 2 & n \text { odd and } r=(n+1) / 2 \\ n & \text { otherwise. }\end{cases}
$$

In this case, there are no repeated eigenvalues, so any rank drop in the $r$-observability matrix is dictated by the presence of zeros in the eigenvectors of the Laplacian. Combining our analysis with a result from Parlangeli and Notarstefano [44], we conclude that for path graphs,

$$
\operatorname{rank}\left(\mathcal{O}_{r}\right) \neq m_{r}^{*} \Longleftrightarrow n-r=(r-1) \bmod p,
$$

where $p$ is an odd prime dividing $n$. For example, the path graph for $n=6$ is not observable for $r=2$ or $r=5$ since $6-$ $2=(2-1) \bmod p$ with $p=1$, and $6-5=(5-1) \bmod p$ for $p=3$.

\section{B. The mEEP with respect to a node and node observability}

We now consider the relationship between the dimensionality of the $r$-observable subspace $\operatorname{rank}\left(\mathcal{O}_{r}\right)$ and the mEEP with respect to node $r$.
First, let $\pi$ be a generic EEP with $m$ cells defined by the indicator matrix $P \in \mathbb{R}^{n \times m}$ and consider the cell-averaging operator $P\left(D^{\pi}\right)^{-1} P^{T}$, as described in Eqs. (10) and (22). It follows from Eqs. (4) and (23) that

$$
\begin{aligned}
\mathcal{O}_{r}\left(P\left(D^{\pi}\right)^{-1} P^{T}\right) & =\left[\begin{array}{c}
\mathbf{e}_{r}^{T}\left(P\left(D^{\pi}\right)^{-1} P^{T}\right) \\
\mathbf{e}_{r}^{T}\left(P\left(D^{\pi}\right)^{-1} P^{T}\right)(-L) \\
\mathbf{e}_{r}^{T}\left(P\left(D^{\pi}\right)^{-1} P^{T}\right)(-L)^{2} \\
\vdots \\
\mathbf{e}_{r}^{T}\left(P\left(D^{\pi}\right)^{-1} P^{T}\right)(-L)^{n-1}
\end{array}\right] \\
& =\frac{1}{\left|C_{k}\right|} \sum_{j \in C_{k}} \mathcal{O}_{j},
\end{aligned}
$$

where $C_{k}$ is the cell in which the node $r$ belongs, and Eq. (32) follows from

$$
\mathbf{e}_{r}^{T}\left(P\left(D^{\pi}\right)^{-1} P^{T}\right)=\frac{1}{\left|C_{k}\right|} \sum_{j \in C_{k}} \mathbf{e}_{j}^{T}
$$

Now consider $\pi_{r}$, an EEP with respect to node $r$. From Eq. (32) we have

$$
\mathcal{O}_{r}\left(P\left(D^{\pi_{r}}\right)^{-1} P^{T}\right)=\mathcal{O}_{r}
$$

Due to the structure of the cell-averaging operator $P\left(D^{\pi_{r}}\right)^{-1} P^{T}$, Eq. (33) implies that $\mathcal{O}_{r}$ has repeated columns for the nodes of each cell. The repetition of columns also means that

$$
\operatorname{rank}\left(\mathcal{O}_{r}\right)=\operatorname{rank}\left(\mathcal{O}_{r} P\right)
$$

since multiplication by $P$ sums up the (repeated) columns in each cell, thus maintaining the rank.

Now consider the consensus dynamics taking place on the quotient graph given by Eq. (13) and, similarly to Eq. (26), define the observability with respect to cell $k$ :

$$
\mathcal{O}_{k}^{\pi}=\left[\begin{array}{c}
\mathbf{e}_{k}^{T} \\
\mathbf{e}_{k}^{T}\left(-L^{\pi}\right) \\
\mathbf{e}_{k}^{T}\left(-L^{\pi}\right)^{2} \\
\vdots \\
\mathbf{e}_{k}^{T}\left(-L^{\pi}\right)^{m-1}
\end{array}\right] \in \mathbb{R}^{m \times m},
$$

where $\mathbf{e}_{k} \in \mathbb{R}^{m}$ is the unit vector in the Cartesian basis with a one in the $k$ th position and zeros elsewhere.

It then follows that the rank of the observability matrix of the full graph with respect to node $r$ is equal to that of the quotient graph with respect to the cell $k$ that contains node $r$. To see this, let node $r$ be in cell $k$. Then $\mathbf{e}_{k}=P^{T} \mathbf{e}_{r}$ and we 
have

$$
\begin{aligned}
\mathcal{O}_{r} P= & {\left[\begin{array}{c}
\mathbf{e}_{r}^{T} P \\
\mathbf{e}_{r}^{T}(-L) P \\
\mathbf{e}_{r}^{T}(-L)^{2} P \\
\vdots \\
\mathbf{e}_{r}^{T}(-L)^{n-1} P
\end{array}\right]=\left[\begin{array}{c}
\mathbf{e}_{k}^{T} \\
\mathbf{e}_{k}^{T}\left(-L^{\pi_{r}}\right) \\
\mathbf{e}_{k}^{T}\left(-L^{\pi_{r}}\right)^{2} \\
\vdots \\
\mathbf{e}_{k}^{T}\left(-L^{\pi_{r}}\right)^{n-1}
\end{array}\right] } \\
= & {\left[\begin{array}{c}
\mathcal{O}_{k}^{\pi_{r}} \\
\mathbf{e}_{k}^{T}\left(-L^{\pi_{r}}\right)^{m} \\
\vdots \\
\mathbf{e}_{k}^{T}\left(-L^{\pi_{r}}\right)^{n-1}
\end{array}\right] . }
\end{aligned}
$$

$$
\mathcal{O}_{4}=\left[\begin{array}{rrrrrrrr}
0 & 0 & 0 & 1 & 0 & 0 & 0 & 0 \\
1 & 1 & 1 & -5 & 1 & 1 & 0 & 0 \\
-6 & -6 & -6 & 30 & -8 & -8 & 2 & 2 \\
36 & 36 & 36 & -184 & 58 & 58 & -20 & -20 \\
-220 & -220 & -220 & 1144 & -398 & -398 & 156 & 156 \\
1364 & 1364 & 1364 & -7176 & 2650 & 2650 & -1108 & -1108 \\
-8540 & -8540 & -8540 & 45272 & -17342 & -17342 & 7516 & 7516 \\
53812 & 53812 & 53812 & -286664 & 112330 & 112330 & -49716 & -49716
\end{array}\right],
$$

which has repeated columns within the cells of $\pi_{4}^{*}=$ $\{\{1,2,3\},\{4\},\{5,6\},\{7,8\}\}$, such that $\operatorname{rank}\left(\mathcal{O}_{4}\right)=4$. The quotient observability matrix for cell $k=2$ (in which node 4 belongs) is given by

$$
\mathcal{O}_{2}^{\pi_{4}^{*}}=\left[\begin{array}{rrrr}
0 & 1 & 0 & 0 \\
3 & -5 & 2 & 0 \\
-18 & 30 & -16 & 4 \\
108 & -184 & 116 & -40
\end{array}\right],
$$

and we have $\operatorname{rank}\left(\mathcal{O}_{4}\right)=\operatorname{rank}\left(\mathcal{O}_{2}^{\pi_{4}^{*}}\right)=m_{4}^{*}=4$. In this case, the upper bound is tight.

The upper bound in Eq. (37) has been explored in relation to graph controllability $[4,34,45]$. In some instances, it can be shown that the bound is tight, i.e., the size of the mEEP $m_{r}^{*}$ is guaranteed to be equal to the dimensionality of the $r$ observable subspace. For example, this is the case for distance regular graphs, for which it is known that $\left|\pi_{r}^{*}\right|=\left|\pi_{D_{r}}\right|$, where the distance partition $\pi_{D_{r}}$ groups nodes with the same shortest path length to node $r$ [45]. Since it is also known that $\left|\pi_{D_{r}}\right| \leqslant$ $\operatorname{rank}\left(\mathcal{O}_{r}\right)$, this implies then that $\left|\pi_{D_{r}}\right|=\operatorname{rank}\left(\mathcal{O}_{r}\right)=\left|\pi_{r}^{*}\right|$ for distance regular graphs. Examples of distance regular graphs include

(1) hypercubes of size $n=2^{d}$ have $m_{r}^{*}=d+1$;

(2) cycles of size $n$ have $m_{r}^{*}=n-\left\lfloor\frac{n-1}{2}\right\rfloor$, as seen in Fig. 6(b).
From the Cayley-Hamilton theorem,

$$
\operatorname{rank}\left(\mathcal{O}_{r} P\right)=\operatorname{rank}\left(\mathcal{O}_{k}^{\pi_{r}}\right),
$$

and together with Eq. (34) we conclude

$$
\operatorname{rank}\left(\mathcal{O}_{r}\right)=\operatorname{rank}\left(\mathcal{O}_{k}^{\pi_{r}^{*}}\right) \leqslant m_{r}^{*},
$$

where $m_{r}^{*}=\left|\pi_{r}^{*}\right|$ is the size of the mEEP with respect to node $r$. Equation (37) states that the rank of the observability of a node under consensus dynamics is equal to the rank of the observability of its cell in the consensus dynamics of the corresponding quotient graph, and the size of the mEEP provides an upper bound for the dimensionality of the $r$-observable subspace.

Example 1 continued. For the graph in Fig. 1(a), the observability matrix with respect to node $r=4$ is

In such cases, obtaining the mEEP provides a graphtheoretical method to evaluate directly the node observability of the consensus dynamics.

However, the upper bound in Eq. (37) is not always tight. Our analysis, i.e., Eq. (37), establishes that $m_{r}^{*}$ is equal to the rank of the observability matrix of the full graph if and only if the corresponding quotient observability matrix is full rank, i.e., if the quotient consensus dynamics is $k$ observable. Importantly, the rank of the quotient observability matrix can also be characterized using Eq. (27), which, as stated above, applies to any diagonalizable Laplacian, even if it is nonsymmetric. In particular, the Laplacian of the quotient graph $L^{\pi}$ is guaranteed to be diagonalizable due to the inheritance of the spectrum from the full Laplacian $L$. Hence,

$$
\operatorname{rank}\left(\mathcal{O}_{k}^{\pi_{r}}\right)=\left|S^{\pi_{r}}\right|-\left|V_{k}^{\pi_{r}}\right|,
$$

where $\left|S^{\pi_{r}}\right|$ is the number distinct eigenvalues of $L^{\pi_{r}}$ and $\left|V_{k}^{\pi_{r}}\right|$ is the number of eigenvalues with an associated subspace that does not span the $k$ th component of the Laplacian of the quotient graph. The failure of the upper bound provided by $m_{r}^{*}$ to be tight originates in the fact that the quotient graph might have repeated eigenvalues or that some of its eigenvectors might have zero components, as we illustrate now with two examples.

Example 1 continued. In our main example in Fig. 1(a), we see from (2) and (29) that $m_{r}^{*} \neq \operatorname{rank}\left(\mathcal{O}_{r}\right)$ for $r=7,8$. As 


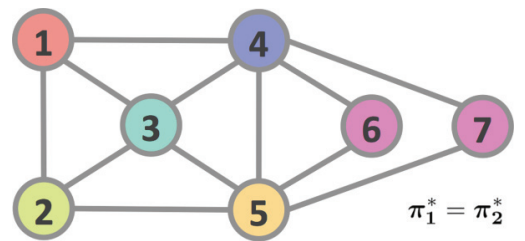

FIG. 7. (Color online) The mEEP of this graph with respect to node 1 (or equivalently node 2 ) indicated with different colors for each cell, $\pi_{1}^{*}=\pi_{2}^{*}=\{\{1\},\{2\},\{3\},\{4\},\{5\},\{6,7\}\}$. For this graph, the dimension of the observable subspace for node 1 (or node 2) is not equal to the size of the corresponding mEEP due to the inheritance of rank-reducing zeros in the eigenvectors of the quotient Laplacian as seen in Eq. (39).

shown in Fig. 1(b), the mEEP of this graph with respect to node $r=7$ has size $m_{7}^{*}=5$. The spectrum of the corresponding quotient graph is $\sigma\left(L^{\pi_{7}^{*}}\right)=\{0,0.6,4,4,6.4\}$, and the inheritance of the repeated eigenvalue 4 by the quotient Laplacian means that, according to Eq. (38), $\operatorname{rank}\left(\mathcal{O}_{7}\right)=4<m_{7}^{*}=5$. The same applies to $\pi_{8}^{*}$.

The other reason for the upper bound in Eq. (37) not to be tight is when rank-reducing zeros in the eigenvectors are transmitted to the quotient Laplacian. For example, consider the graph in Fig. 7 and its mEEP with respect to node $r=1: \pi_{1}^{*}=\{\{1\},\{2\},\{3\},\{4\},\{5\},\{6,7\}\}$ such that $m_{1}^{*}=6$. The eigenvalues of the quotient Laplacian are distinct: $\sigma\left(L^{\pi_{1}^{*}}\right)=\{0,1.5858,3.5858$, $4.4142,6.4142,6\}$ with eigenvectors given by the columns of

$$
U^{\pi_{1}^{*}}=\left[\begin{array}{rrrrrr}
1 & 1.2718 & -1.6002 & 0.9798 & 0.6628 & 0 \\
1 & 1.2718 & 1.6002 & 0.9798 & -0.6628 & 0 \\
1 & 0.8149 & 0.0000 & -1.7476 & -0.0000 & -1.3587 \\
1 & -0.2881 & -0.6628 & -0.6179 & -1.6002 & 1.3587 \\
1 & -0.2881 & 0.6628 & -0.6179 & 1.6002 & 1.3587 \\
1 & -1.3911 & 0 & 0.5119 & 0 & -0.6794
\end{array}\right]
$$

Note that $\mathbf{u}_{6}^{\pi}$ has a zero in cell $k=1$ and $\operatorname{rank}\left(\mathcal{O}_{1}^{\pi_{1}^{*}}\right)=5 \neq m_{1}^{*}$, from Eq. (38). The same applies to node $r=2$.

\section{DISCUSSION AND CONCLUSIONS}

In this paper, we have studied the intrinsic relationship between graph structure, as revealed by the external equitable partition; the spectral characteristics of the associated graph Laplacian; and the dynamical implications for linear network consensus. In particular, the relationship between consensus dynamics on a graph and its EEP highlights how this partition captures graph symmetries that induce time invariances in the dynamics. Such symmetries may be eliminated or reduced via the construction of the quotient graph and lead to a related coarse-grained consensus dynamics taking place on the quotient graph of the EEP. It is important to mention that although the quotient graph is actually a directed graph, it has particular properties (strong connectedness, real eigenvalues) due to the direct inheritance of such properties from the original graph.

We have also studied how certain spectral and graph theoretical features of the EEP with respect to a single node allow us to characterize the convergence and observability of the dynamics for each node, i.e., nodes exhibiting a nontrivial EEP can, in some cases, exhibit faster local convergence compared to the global convergence for the graph. Faster local convergence is guaranteed when the second largest eigenvalue of the quotient graph is larger than that of the full graph or, in other words, when the first nonzero eigenvalue is not "inherited" by the quotient graph.

On the other hand, we have also shown that the observability of the full dynamics with respect to each node is determined by the observability of the quotient Laplacian dynamics with respect to its corresponding EEP cell. Hence, the size of the mEEP with respect to a node provides an upper bound on the dimension of the corresponding observable subspace. However, our precise spectral characterization of the dimension of the observable subspace shows how the transmission to the quotient graph Laplacian of eigenvalue degeneracies or of rank-reducing zero components (leading to repeated columns in the observability matrix) can make this bound not tight. Hence the existence of a nontrivial mEEP is a necessary condition for the coarse-grained dynamics given by the mEEP to converge faster. In general, the implication is that there is a link between partial observability from a node and the possibility that the node will converge faster.

The increased understanding of the spectral conditions under which the EEP may be used to describe a reduced system allows us to analyze and design networks with optimized features, that can ensure, for example, fast convergence or observability. In this respect, a possible avenue of future research is the optimization of edge weights such that, for example, the convergence rate for a particular node could be maximized, or the observability rank minimized for the prediction of the final consensus value based on few values of the history of the node [30]. Another direction worth exploring is the extension of our results to general directed graphs, for which the Laplacian is not symmetric. Future work also includes the characterization of the external equitable partition for classes of constructive and randomly generated graphs, and in particular the classification of "knowledgeable" or "influential" nodes in terms of their dynamical convergence and observability properties.

\section{ACKNOWLEDGMENTS}

N.O'C. was funded by a Wellcome Trust Doctoral Studentship. Y.Y. acknowledges support from the Microsoft Research Cambridge Ph.D. Scholarship program. G.-B.S. acknowledges the support of the EPSRC Centre for Synthetic Biology and Innovation (Project No. EP/G036004/1) at Imperial. M.B. acknowledges support from EPSRC Grant No. EP/I032223/1 and EPSRC Grant No. EP/I017267/1 under the Mathematics Underpinning the Digital Economy program.

\section{APPENDIX: ALGORITHM TO COMPUTE THE mEEP WITH RESPECT TO A SINGLE NODE}

We now present an efficient method to compute the minimal external equitable partition with respect to a node. This algorithm is inspired by partition refinement algorithms used to 
find graph automorphisms [33]. A related but distinct approach was outlined separately by Cao et al. [34].

Briefly, our algorithm proceeds as follows:

(1) Initialization. Generate a $k=2$ partition with the chosen node in one cell and all other nodes in the second cell.

(2) Iteration. Build the profile matrix $N \in R^{n \times k}$ for current partition of size $k$, such that $N_{i j}$ is the out-degree from node $i$ to nodes in cell $j$ for $i \notin C_{j}$, and $N_{i j}=0$ for $i \in C_{j}$. Group identical node profiles (rows of $N$ ) within any single cell and split the cell accordingly to get a refined partition with an increased number of cells. Return to Step 2.

(3) Termination. When all rows within each cell are the same, no further splits are needed. The mEEP has been found.
By construction, the algorithm finds an EEP. To see that we find the minimal EEP, consider whether the algorithm could result in a cell being split which could remain un-split for a coarser EEP. According to our algorithm, a cell $C_{1}$ is split if two nodes in $C_{1}$ have differing out-degree to another cell, e.g., $C_{2}$. If $C_{2}$ were split later becoming $C_{3}$ and $C_{4}$, it follows from the algorithm that any split of $C_{2}$ conserves the same nodes divided between the new cells $C_{3}$ and $C_{4}$, and hence the total out-degree. Therefore, any re-distribution of the out-degree between the new cells cannot result in equivalent profiles for an upstream node pair in $C_{1}$. If we apply this logic inductively, we see that the algorithm results in the minimal (unique) EEP with respect to the node.
[1] D. J. Watts and S. H. Strogatz, Nature (London) 393, 440 (1998).

[2] M. Barahona and L. M. Pecora, Phys. Rev. Lett. 89, 054101 (2002).

[3] Y.-Y. Liu, J.-J. Slotine, and A.-L. Barabási, Nature (London) 473, 167 (2011).

[4] M. Egerstedt, S. Martini, M. Cao, K. Camlibel, and A. Bicchi, IEEE Control Systems 32, 66 (2012).

[5] L. Xiao, S. Boyd, and S. Lall, in IEEE Fourth International Symposium on Information Processing in Sensor Networks (IEEE, Piscataway, 2005), pp. 63-70.

[6] U. Krause, Modellierung und Simulation von Dynamiken mit vielen interagierenden Akteuren (Bremen University, Bremen, 1997).

[7] V. Sood and S. Redner, Phys. Rev. Lett. 94, 178701 (2005).

[8] H. Tanner, A. Jadbabaie, and G. Pappas, Proceedings of the 42nd IEEE Conference on Decision and Control 2010 (IEEE, Piscataway, 2003).

[9] T. Vicsek, A. Czirók, E. Ben-Jacob, I. Cohen, and O. Shochet, Phys. Rev. Lett. 75, 1226 (1995).

[10] A. Jadbabaie, J. Lin, and A. Morse, IEEE Transactions on Automatic Control 48, 988 (2003).

[11] L. M. Pecora and M. Barahona, Chaos and Complexity Letters 1, 61 (2005).

[12] R. Olfati-Saber, J. Fax, and R. Murray, in Proceedings of the IEEE, Vol. 95 (IEEE, Piscataway, 2007), pp. 215-233.

[13] R. Olfati-Saber and R. Murray, IEEE Transactions on Automatic Control 49, 1520 (2004).

[14] V. Blondel, J. Hendrickx, A. Olshevsky, and J. Tsitsiklis, in 44th IEEE Conference on Decision and Control (IEEE, Piscataway, 2005), pp. 2996-3000.

[15] R. Olfati-Saber, IEEE Transactions on Automatic Control 51, 401 (2006).

[16] W. Ren, R. W. Beard, and E. M. Atkins, in IEEE American Control Conference (IEEE, Piscataway, 2005), pp. 1859-1864.

[17] D. Kempe, A. Dobra, and J. Gehrke, 44th Annual IEEE Symposium on Foundations of Computer Science (IEEE, Piscataway, 2003), pp. 482-491.

[18] J.-C. Delvenne, S. Yaliraki, and M. Barahona, Proc. Natl. Acad. Sci. U.S.A. 107, 12755 (2010).

[19] R. Lambiotte, R. Sinatra, J.-C. Delvenne, T. S. Evans, M. Barahona, and V. Latora, Phys. Rev. E 84, 017102 (2011).
[20] S. Gómez, A. Diaz-Guilera, J. Gómez-Gardenes, C. J. PérezVicente, Y. Moreno, and A. Arenas, Phys. Rev. Lett. 110, 028701 (2013).

[21] P. J. Menck, J. Heitzig, N. Marwan, and J. Kurths, Nat. Phys. 9, 89 (2013).

[22] D. L. Powers and M. S. Mohammad, Linear Algebra and its Applications 48, 145 (1982).

[23] B. McKay, in Proceedings of Conference on Combinatorial Mathematics (Springer, Berlin, 1978), pp. 223-232.

[24] S. Martini, M. Egerstedt, and A. Bicchi, Int. J. Syst., Control Commun. 2, 100 (2010).

[25] A. Rahmani, M. Ji, M. Mesbahi, and M. Egerstedt, SIAM J. Control Optim. 48, 162 (2009).

[26] D. M. Cardoso, C. Delorme, and P. Rama, Eur. J. Combinatorics 28, 665 (2007).

[27] K. Ogata, Modern Control Engineering, 5th ed. (Prentice Hall, Upper Saddle River, 2009).

[28] Y.-Y. Liu, J.-J. Slotine, and A.-L. Barabási, Proc. Natl. Acad. Sci. U.S.A. 110, 2460 (2013).

[29] S. Sundaram and C. N. Hadjicostis, in Proceedings of the 6th International Conference on Information Processing in Sensor Networks (ACM Press, New York, 2007), pp. 99108.

[30] Y. Yuan, G.-B. Stan, L. Shi, M. Barahona, and J. Goncalves, Automatica 49, 1227 (2013).

[31] B. Mohar, Graph theory, Combinatorics, and Applications 2, 871 (1991).

[32] C. Godsil, Algebraic Combinatorics (Chapman Hall/CRC, London, 1993).

[33] G. Hahn and G. Sabidussi, Graph Symmetry: Algebraic Methods and Applications, NATO ASI Series. Series C, Mathematical and Physical Sciences, Vol. 497 (Kluwer Academic Publishers, Dordrecht, 1997).

[34] M. Cao, S. Zhang, and M. Camlibel, IEEE Transactions on Automatic Control 58, 465 (2013).

[35] F. Gantmacher, The Theory of Matrices, Vol. 1 (American Mathematical Society, Providence, 1959).

[36] L. Xiao and S. Boyd, Systems and Control Letters 53, 65 (2004).

[37] W. Lu, B. Liu, and T. Chen, Chaos 20, 013120 (2010).

[38] G. Russo and Jean-Jacques E. Slotine, Phys. Rev. E 84, 041929 (2011). 
[39] A. Jadbabaie, N. Motee, and M. Barahona, American Control Conference 5, 4296 (2004).

[40] A. E. Brouwer, A. M. Cohen, and A. Neumaier, DistanceRegular Graphs (Springer-Verlag, New York, 1989).

[41] D. Spielman, Lecture Notes - Spectral Graph Theory (2012).

[42] S. Sundaram and C. Hadjicostis, in Proceedings of the IEEE American Control Conference (IEEE, Piscataway, 2007), pp. 711-716.
[43] D. Boley, T. Franklin, and D. Vandevoorde, Scientific Computing 27 (1998).

[44] G. Parlangeli and G. Notarstefano, in Proceedings of the 49th IEEE Conference on Decision and Control (IEEE, Piscataway, 2010), pp. 1492-1497.

[45] S. Zhang, M. Camlibel, and M. Cao, in Proceedings of the 50th IEEE Conference on Decision and Control and European Control Conference (IEEE, Piscataway, 2011), pp. 759-764. 